\title{
The Orion Nebula in the Far-Infrared: High-J CO and fine-structure lines mapped by FIFI-LS/SOFIA
}

\author{
Randolf Klein ${ }^{1}$, Leslie W. Looney ${ }^{2}$, Erin Cox $^{2}$, Christian Fischer ${ }^{3}$, \\ Christof Iserlohe $^{3}$, Alfred Krabbe ${ }^{3}$ \\ ${ }^{1}$ SOFIA-USRA, NASA Ames Research Center, MS 232-12, PO box 1, Moffet Field, CA 94035 \\ email: rklein@sofia.usra.edu \\ ${ }^{2}$ University of Illinois, Dept. of Astronomy, MC-221, 1002 W. Green St., Urbana, IL 61801 \\ Deutsches SOFIA Institut, Pfaffenwaldring 29, D-70569 Stuttgart
}

\begin{abstract}
The Orion Nebula is the closest massive star forming region allowing us to study the physical conditions in such a region with high spatial resolution. We used the far infrared integral-field spectrometer, FIFI-LS, on-board the airborne observatory SOFIA to study the atomic and molecular gas in the Orion Nebula at medium spectral resolution.

The large maps obtained with FIFI-LS cover the nebula from the BN/KL-object to the bar in several fine structure lines. They allow us to study the conditions of the photon-dominated region and the interface to the molecular cloud with unprecedented detail.

Another investigation targeted the molecular gas in the BN/KL region of the Orion Nebula, which is stirred up by a violent explosion about 500 years ago. The explosion drives a wide angled molecular outflow. We present maps of several high-J CO observations, allowing us to analyze the heated molecular gas.
\end{abstract}

Keywords. stars: formation, infrared: ISM, ISM: Orion Nebula

\section{Preliminary Results}

The observations were taken during the commissioning of FIFI-LS (Klein et al. 2014) on board of NASA's airborne observatory SOFIA (Young et al. 2012) last year and as recent as March 2015. The data reduction and calibration is still under development. Mainly, the atmospheric absorption correction and flux calibration is still in works.

The results of the fine structure line observations of [CII] and [OI] are shown in Fig. 1. Next to the line emission, a FIFI-LS observation also yields the underlying continuum. The continuum emission is peaking in the $\mathrm{BN} / \mathrm{KL}$ object, where deeply embedded protostars heat the dust (De Buizer et al. 2012). The dust east of the Trapezium is the second peak. The Bar is not very prominent in the dust continuum. However, the [CII] emission clearly traces the Orion Bar, and the north-eastern wall of the cavity, which is cleared out by the trapezium stars. The emission comes from the Photon-Dominated-Region (PDR), where the UV-radiation from the Trapezium Cluster irradiates the surrounding molecular gas.

Figure 1 also shows the [OI] lines, which trace the Bar like the [CII] lines, as these finestructure lines are the main cooling lines in a PDR. When the flux calibration is done, we can start interpreting the line ratios in term of density, temperature, and excitation conditions. Since the Bar is a nearly edge-on PDR, the width and position of the line emission can indicate how far the UV can penetrate. 

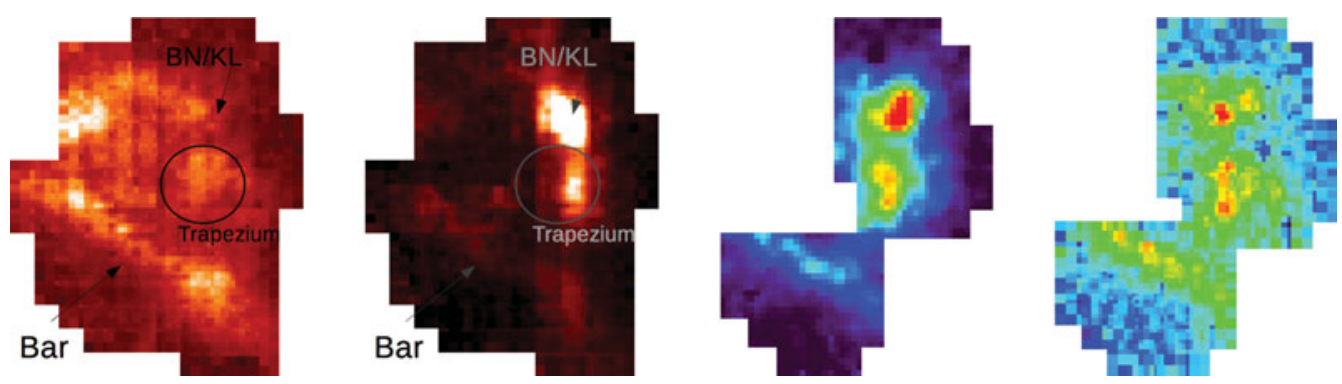

Figure 1. From left to right: [CII] $158 \mu \mathrm{m}$ line and continuum emission, [OI] $63 \mu \mathrm{m}$ and [OI] $145 \mu \mathrm{m}$ line emission
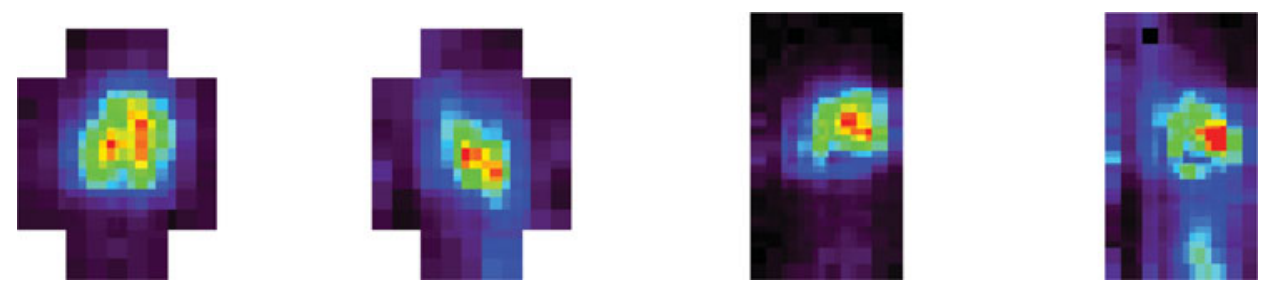

Figure 2. High J-CO from the BN/KL-object lines from left to right: $J=16 \rightarrow 15$ at $163 \mu \mathrm{m}$, continuum at $163 \mu \mathrm{m}, J=14 \rightarrow 13$ at $186 \mu \mathrm{m}$, continuum at $186 \mu \mathrm{m}$

With FIFI-LS, we also detected the high-J CO lines in the Becklin-Neugebauer/ Kleinmann-Low (BN/KL) object reported by Goicoechea et al. (2015a). Figure 2 shows the two lobes of the wide molecular outflow caused by an explosions about 500 years ago.

\section{Outlook}

The next step will be to calibrate the FIFI-LS data and cross calibrate the results with existing observations like Goicoechea et al. (2015b). After that a quantitative analysis can follow. We will look at the fine structure line intensities and compare them to PDR models like Pound \& Wolfire (2008) to drive the physical conditions in the PDRs in the Orion Nebula and how the parental could is affected by the forming stars. Similarly, the study of the warm gas in BN/KL via the high-J CO lines will help to understand the effects of star formation on the surrounding molecular cloud. The large maps by FIFI-LS of this well studied region will demonstrate the mapping capabilities of FIFI-LS on board SOFIA.

\section{References}

De Buizer, J. M., Morris, M. R., Becklin, E. E., Zinnecker, H., Herter, T. L., Adams, J. D., Shuping, R. Y., \& Vacca, W. D. 2012, ApJ, 749, L23

Goicoechea, J. R., Chavarría, L., Cernicharo, J., Neufeld, D. A., Vavrek, R., Bergin, E. A., Cuadrado, S., Encrenaz, P., Etxaluze, M., Melnick, G. J., \& Polehampton, E. 2015a, ApJ, 799,102

Goicoechea, J. R. et al. 2015b, ArXiv e-prints

Klein, R., Beckmann, S., Bryant, A., Colditz, S., Fischer, C., Fumi, F., Geis, N., Hönle, R., Krabbe, A., Looney, L., Poglitsch, A., Raab, W., Rebell, F., \& Savage, M. 2014, in Society of Photo-Optical Instrumentation Engineers (SPIE) Conference Series, Vol. 9147, 2

Pound, M. W. \& Wolfire, M. G. 2008, in ASP Conference Series, Vol. 394, Astronomical Data Analysis Software and Systems XVII, ed. R. W. Argyle, P. S. Bunclark, \& J. R. Lewis, 654

Young, E. T. et al. 2012, ApJ, 749, L17 